\title{
Research on rendering speed based on Maya and mental ray
}

\author{
Yang Gao \\ South China university of technology, China \\ *gaoyangyang100@gmail.com
}

\section{Keywords: MAYA; Mental Ray; agency rendering; speed}

\begin{abstract}
In order to improve the multimedia animation rendering speed, for the purpose of aiming at the shortcomings of the Maya bring renderer speed slow, from the viewpoint of "agency rendering", combining with mental ray using the geometry shader, from the material of model, the surface model,comparative study of different renderer on the same surface material model rendering speed and using a proxy model and using the rendering speed of the agent model, methods to enhance the rendering speed is achieved through the actual operation.
\end{abstract}

\section{Introduction}

Image generated by the software in the computer graphics process model is the process of rendering. In MAYA animation process, it is one of the most time consuming process, is to create the computer graphics the last of the key steps. The speed is not totally depends on the condition of producer proficiency,more is dependent on the performance of the machine. For now, the mainstream renderer Mental Ray was introduced in the MAYA design software, provides superior to bring their own renderer for MAYA and other renderers work efficiency and quality. At the same time, MAYA for mental ray provides considerable development space. A combination of both, foster strengths and circumvent weaknesses and better play to their advantages.

"Agency rendering" is one of the more commonly used nowadays rendering speed method. Its main principle is through the agency of a simple object to invoke another complex external model. Therefore, on the rendering speed, experimental platform for MAYA play the advantages of Mental Ray, using Geometry Shader, get a way of speed, and is verified by actual case analysis.

\section{Theoretical Analysis}

Digital animation as one of the branch of computer art, it is art fusion of art and computer graphics, animation, is built on both a new art form, and become the pioneer of modern multimedia animation development mainstream. In the past, this kind of technology is mainly used in the military field, but with the growing popularity of the science and technology to the development of computer, digital art develops slowly refined by now become an independent subject design every aspect of life, such as UI design, graphic design, industrial design, etc.

In the current market is used to implement digital video and 3D animation tools, MAYA has become the preferred solution. The generation of a 3D animation need to experience model, texture, lighting layout, bones, binding to the final rendering output this several stages. Industry there is a saying called: "Depend on the material make up the defects of the model, rely on light to make up for the lack of material, by rendering to compensate for bad light." That argument is not do not take the lighting model material, but to highlight as the final rendering images of the importance of the render link.So in the case of limited hardware conditions, in order to shorten the rendering time, measures taken are: can often reduce the image quality, improve the computer configuration, the rational allocation of render parameters, etc. Maximum use of existing computer rendering task performance. This method less input costs, suitable for most animators. Beyond that is used for the renderer, such as Mental Ray. It is engaged in the design and animation industry personnel of one of the necessary software. Ray as apply colours to a drawing class, and getting the tip of the plugin was introduced into 
various kinds of design software, such as MAYA,3DMAX, in addition, getting the light Ray tracing function of superb. For now it will play incisively and vividly, ray tracing algorithm can implement other rendering the product it is difficult to achieve real results. Mental Ray is a very complicated programming raytrace renderer, getting the material of Ray model are some of the standard $\mathrm{C}++/$ Clanguage to write the program code, and so on speed is superior to the model of material written in other scripting languages. In addition, it compared with the renderer MAYA bring much faster. agent rendering function is the function of the MAYA did not. It by using the Geometry Shader in rendering by rendering a simple object to implement complex rendering, thus greatly improve the rendering speed.

In the actual operation process, usually measured in number of objects in front of the scene file size.Rendering speed is affected by the number of geometric surface in the space,with the increase of number of geometric surface, slow to render. Not only that, the rendering speed will be affected by camera movement, the scene in the influence of various materials. Typically, scene model and parts are often strict with face. But under the limited number of delicate degree of the model will be greatly decreased, the scene will be delicate and beautiful will also be affected. Can be found in the set such a rule:repeat item of the stack, such as trees, houses, and the window of a large number of use, such as trees, for example, a tree model in the presence of several general about 5000 to 10000 . Except the special demand,can not only a tree in the scene. In such a large number of repeat items will make file increases, when the final render will be greatly dragged down rendering speed,lower efficiency.It also have the fewest number of surface of nearly100000.

To solve such problems, the traditional solution is:layered rendering.Through the scene subdivided into foreground, middle and foreground, main is to use software such as AE post-production for synthesis. But the scene file, the greater the requirements of the layered more careful. In addition, there are some "lazy" small method in detail. Trees, for example, can be used only on the surface of the two cross tree, but only on the surface of the two cross this tree usually applies to far below lens, or relatively static camera. Because of such trees prone to wear in the process of camera movement states to break the surface, and so on and so forth. In addition to using particle replacement, as well as a variety of plug-ins, etc. However, these traditional methods are this or that the defects and limitations, can not real to solve the problem.

In addition to the traditional way, Mental Ray's Geometry Shader might solve this problem: the so-called Geometry in Mental Ray in the definition of the object is at least three vertices. Its main principle is through the use of Geometry Shader to more complex polyhedral model with simple Geometry, so as to greatly improve the speed of rendering.

\section{The Operation}

Take Knight's model for example, to copy it and layer in the scene (Fig. 1), so it can separate for rendering. From the picture shows the surface of the each model number up to three hundred and sixty thousand (Fig. 2).

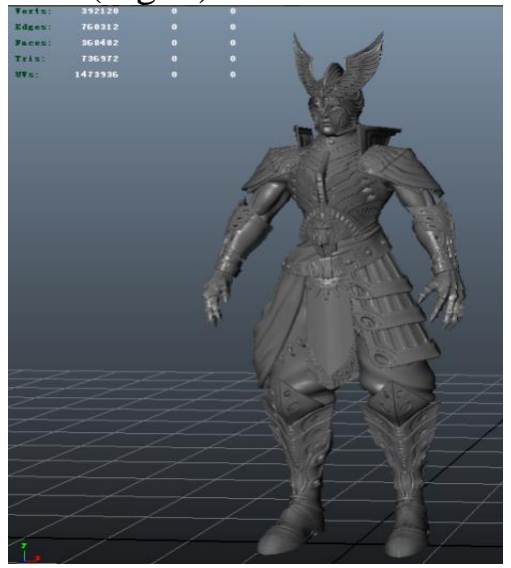

Figure 1. Knight's model and the parameters

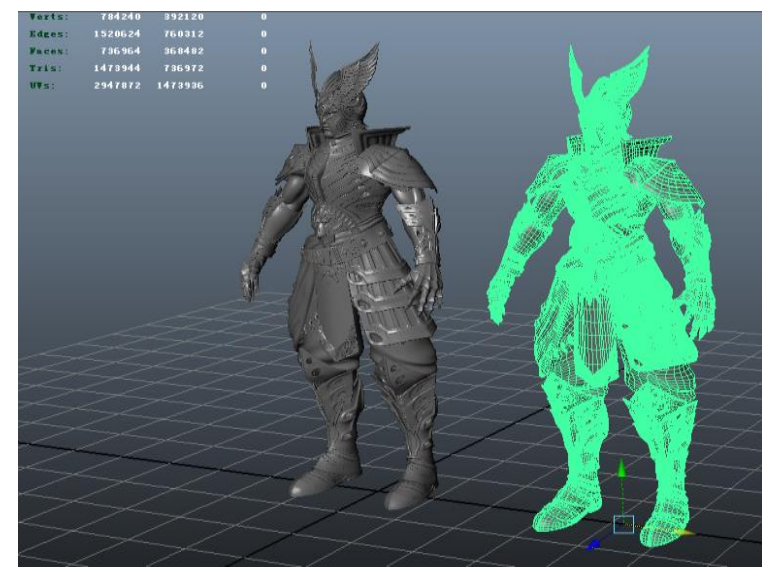

Figure 2. knights model replication 
Create a video camera, and basic on "Three lighting " to ensure the rendering result is not affected by Angle and lighting (Fig. 3). Open the window - rendering editors - Hypershade (HY), under the same external conditions for the two warriors model gives"blinn"shader ball respectively.

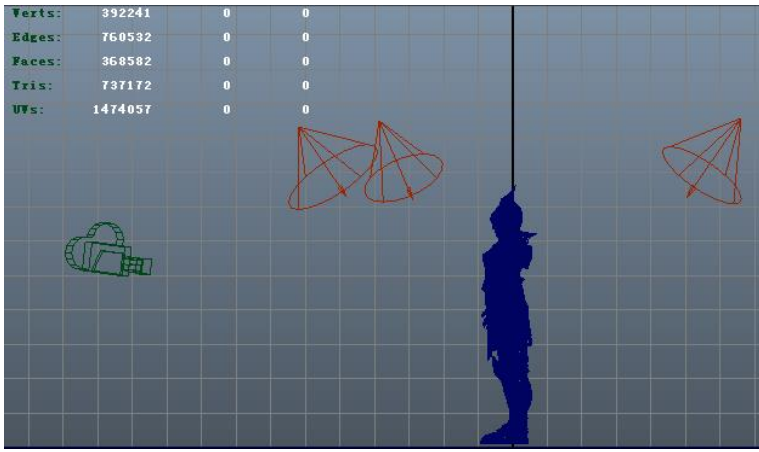

Figure 3. model light camera position

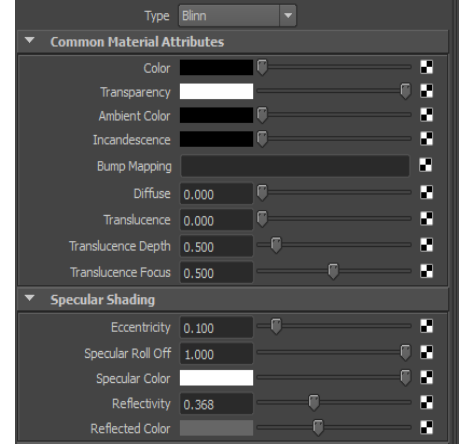

Figure 4. model material properties

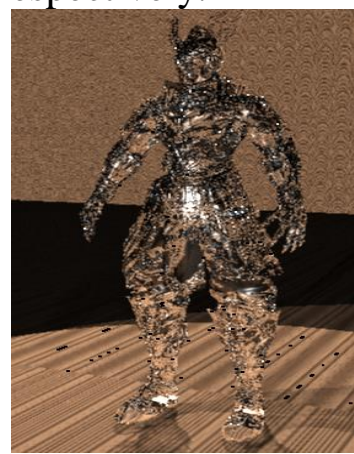

Figure 5. glass material

By changing the material properties of transparency, refraction, reflection, reflection color make the present glass textures (Fig. 4) and a metallic simple sense. Monster closed glass layer, according to open the render settings to confirm render using the default renderer for MAYA Softwear, output option is in the rendering quality model, then the glass up a monster as shown in Fig. 5. By rendering the diagnostic information bar can know the whole rendering process (Fig. 6) is 0.01 seconds, switch to the MAYA bring renderer repeat the rendering process for rendering speed is 0.04 seconds (Fig. 7). The above is only for a single model use different renderers for rendering.

Broker model rendering experiments below: first, to create a BOX as an agent model, and the BOX attributes panel Geometry shader in check the enable Geometry shader create node "mip_binaryproxy", open the node attribute set rendering address after open up a renderer (Fig. 8). From Fig. 9 we can see the simple geometric objects into a complex model, at the same time rendering speed of just 0.01 seconds. In the case of do not use this way, rendering speed of 0.04 seconds, the whole process to improve for 0.03 seconds.

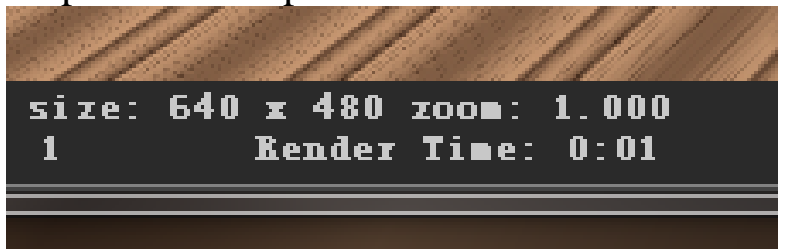

Figure 6. mental ray renderings

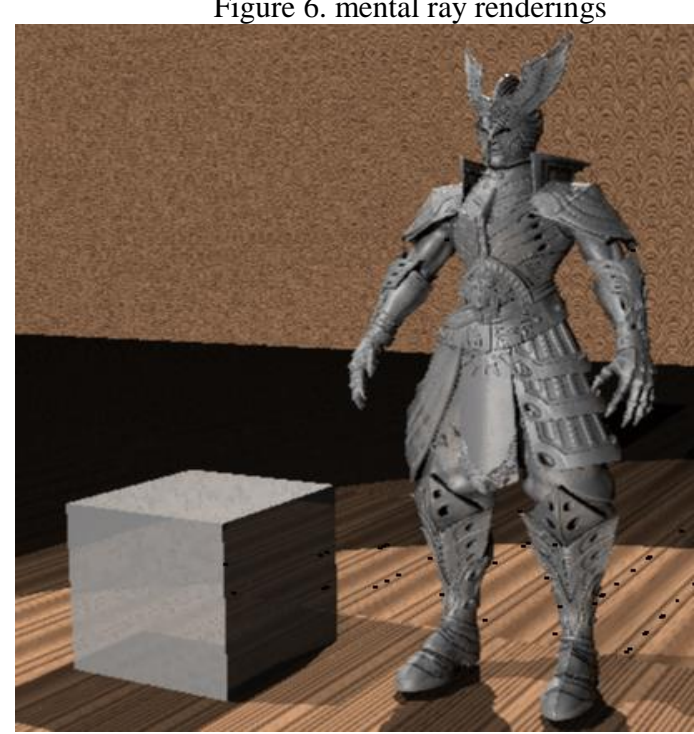

Figure 8. befor agent rendering

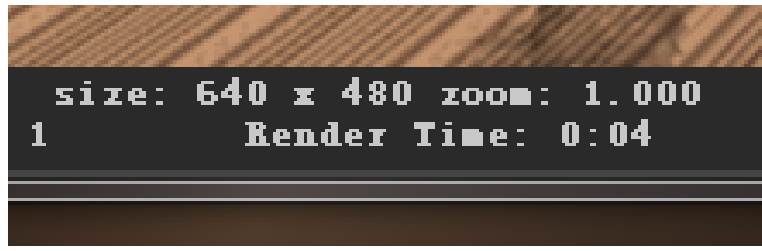

Figure 7. softwear renderings

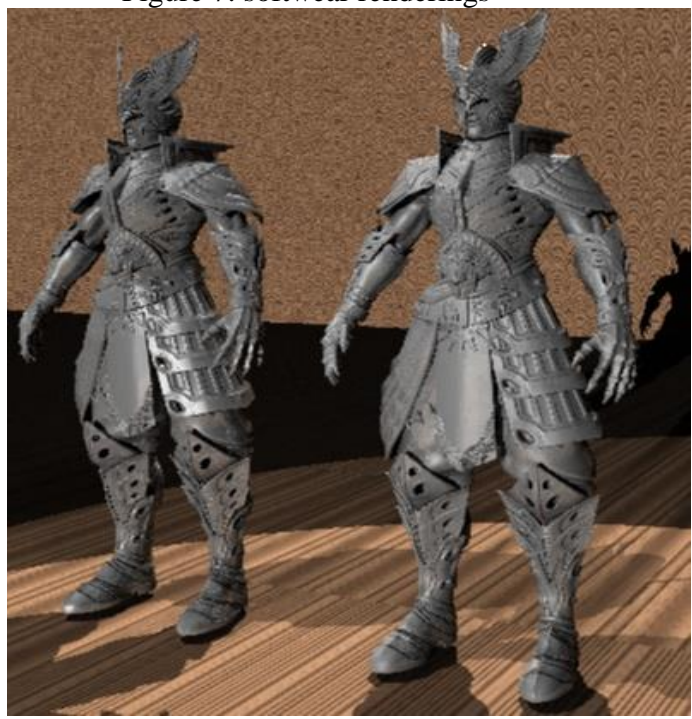

Figure 9. after agent rendering

Through practice can learn Geometry Shader rendering can be replace by rendering a simple object is rendering complex objects. In the whole process, the rendering speed of 0.01 seconds. Got very big improvement. And even Smooth for several times, rendering time is not too much change, this is credit for Geometry Shader. 


\section{Conclusions}

Combination with MAYA and Mental Ray,making it possible to use simple items in the rendering to replace the complex objects, the ability to achieve fast rendering. Not only making smaller file overall capacity, and can complete rendering efficient purpose. This method is easy to reuse, In understanding the principle and fabrication method based on, a little to draw inferences about other cases from one instance to achieve more effect. For example: giving objects of different colors, making different movements and facial expressions, perhaps after all without the use of group animation or animation curve copy, can easily finish pedestrian movement, speeding through traffic tools, or simulate thousands people dogfight scenes. In the subsequent will further practice.

\section{References}

[1] Donald Hearn M.Pauline Baker, Cai Shijie, Song Jiqiang, Cai Min. Computer graphics [M]. Publishing House of electronics industry, 2005

[2] Wang Hao, Liu Kuo.Maya6 Advanced Maya Rendering and rendering technology, the essence of Shading (on). Weapon Industry Press, 2004

[3] Yang Yongtao. Mental Ray for Maya. Weapon Industry Press, 2005

[4] Zhao Guangyu, Guo Tao. The art of rendering. Weapon Industry Press, 2007

[5] Lang Yu. MAYA 6 from entry to the master. China Youth Publishing House, 2005

[6] Zhu Yihao, Li Jutao. Three-dimensional film and TV animation production [M]. Beijing Weapon Industry Press, 2004

[7] Wuhan Sagittarius, Maya6 super manual (3) animation article [M]. Beijing Ordnance Industry Press, 2005

[8] Zhang Xiaowei, Yu Zhong.Maya three-dimensional dynamic world [M]. Beijing ocean publishing house, 2000

[9] Wang Shengwei. 3D animation Maya technology [J]. software guide, 2010

[10] Liu Jun. Based on the Maya and Mental Ray technology of 3d animation [D]. Shanghai Jiao Tong University, 2008 